\title{
Impact of hemoglobin breakdown products in the spectral analysis of burn wounds using spatial frequency domain spectroscopy
}

Rolf B. Saager

Rebecca A. Rowland

Melissa L. Baldado

Gordon T. Kennedy

Nicole P. Bernal

Adrien Ponticorvo

Robert J. Christy

Anthony J. Durkin 


\section{Impact of hemoglobin breakdown products in the spectral analysis of burn wounds using spatial frequency domain spectroscopy}

\author{
Rolf B. Saager, ${ }^{a}$ Rebecca A. Rowland, ${ }^{a}$ \\ Melissa L. Baldado, ${ }^{a}$ Gordon T. Kennedy, ${ }^{a}$ \\ Nicole P. Bernal, ${ }^{b}$ Adrien Ponticorvo, ${ }^{a}$ \\ Robert J. Christy, ${ }^{\mathrm{c}}$ and Anthony J. Durkin ${ }^{\mathrm{a}, \mathrm{d}, *}$ \\ aUniversity of California Irvine, Beckman Laser Institute and \\ Medical Clinic, Irvine, California, United States \\ bUC Irvine Regional Burn Center, Department of Surgery, Orange, \\ California, United States \\ 'United States Army Institute of Surgical Research, Burn and \\ Soft Tissue Injury, San Antonio, Texas, United States \\ dUniversity of California Irvine, Department of Biomedical \\ Engineering, Irvine, California, United States
}

\begin{abstract}
Burn wounds and wound healing invoke several biological processes that may complicate the interpretation of spectral imaging data. Through analysis of spatial frequency domain spectroscopy data (450 to $1000 \mathrm{~nm}$ ) obtained from longitudinal investigations using a graded porcine burn wound healing model, we have identified features in the absorption spectrum that appear to suggest the presence of hemoglobin breakdown products, e.g., methemoglobin. Our results show that the calculated concentrations of methemoglobin directly correlate with burn severity, $24 \mathrm{~h}$ after the injury. In addition, tissue parameters such as oxygenation $\left(\mathrm{StO}_{2}\right)$ and water fraction may be underestimated by $20 \%$ and $78 \%$, respectively, if methemoglobin is not included in the spectral analysis. $\odot$ The Authors. Published by SPIE under a Creative Commons Attribution 4.0 Unported License. Distribution or reproduction of this work in whole or in part requires full attribution of the original publication, including its DOI. [DOI: 10.1117/1.JBO.24.2.020501]
\end{abstract}

Keywords: burns; hemoglobin; methemoglobin; spectroscopy; multispectral imaging; spatial frequency domain spectroscopy.

Paper 180584LR received Oct. 17, 2018; accepted for publication Jan. 15, 2019; published online Feb. 5, 2019.

There are several physiological parameters associated with burn wounds and wound healing that can be detected and quantified optically. Structural changes to tissue from thermal insult (collagen denaturation, protein coagulation, etc.) can be detected by changes in how light scatters through tissue (i.e., reduced scattering coefficient). Similarly, changes in functional characteristics, such as tissue oxygen saturation, blood volume, and water fraction, (edema) can be monitored by how tissue absorbs light (i.e., absorption coefficient). ${ }^{1,2}$ In the case of thermal burn wounds and its subsequent wound healing response, however,

*Address all correspondence to Anthony J. Durkin, E-mail: adurkin@uci.edu there are biological processes (e.g., extravasation, inflammation, granulation, and tissue remodeling $)^{3,4}$ that can affect how spectral imaging methods interpret both structural and functional parameters in tissue. With a thermal insult, there can be disruption of the vascular network, leaving blood trapped within the affected tissue. As trapped hemoglobin breaks down, the byproducts (e.g., methemoglobin) could then contribute to the measured spectra. ${ }^{5}$ This process may have previously uncharacterized consequences for optical imaging methods as it may interfere with standard methods for interpreting and quantifying absorption data in terms of parameters such as tissue oxygen saturation.

While multispectral approaches have been applied toward the early detection and triage of burn wounds and shown great potential, these instruments and approaches often use limited spectral sampling and resolution. ${ }^{1,2,6,7}$ Spatial frequency domain imaging (SFDI) has been of particular interest in this application as it quantifies absorption and scattering optical properties of in-vivo tissue at every wavelength independently using the diffuse reflectance response from tissue as a function of the pattern frequency projected and a scaled Monte Carlo to model photon migration. ${ }^{8}$ This enables separation of changes in tissue structure from those resulting from changes in absorption characteristics without any additional presumed scattering model or chromophores. ${ }^{2,9-13}$ When it comes to determining tissue constituents, many other analysis techniques depend on a priori models that presume all potential sources of optical contrast within tissue in order to interpret measured data. Though blood breakdown products such as methemoglobin are not usually considered in optical device measurements of tissue oxygen saturation, it has already been suggested that they might play a significant role in burn wounds. ${ }^{14,15}$

Our primary objective is to investigate whether spectral signatures from methemoglobin (MetHb) are present, in vivo, in optical measurements of burn wounds and what influence they may exert in the accurate and reliable determination of other tissue functional parameters. Additionally, we will evaluate how these blood breakdown products might impact the performance of a commercial multispectral imager that has coarse spectral resolution and relatively few illumination wavelengths. This is done as a side-by-side comparison with the high spectral resolution approach using the same set of in vivo burn data as described as follows.

In this investigation, spectrally resolved tissue optical properties were calculated using a technique called spatial frequency domain spectroscopy (SFDS). ${ }^{16}$ Here, the reflectance data were measured by a custom-built, handheld spectral imaging system, described in greater detail elsewhere. ${ }^{17}$ This device projects broadband (450 to $1000 \mathrm{~nm}$ ) illumination of sinusoidal intensity and then detects the spatial frequency-dependent responses from tissue along a $\sim 3-\mathrm{cm}$ line, at $\sim 1 \mathrm{~nm}$ spectral resolution. In this study, five evenly spaced spatial frequencies were employed ranging from 0 to $0.2 \mathrm{~mm}^{-1}$. Three phases (i.e., $0 \mathrm{deg}, 120 \mathrm{deg}$, and $240 \mathrm{deg}$ ) of each frequency were projected, as has been our convention, so that the frequency-dependent reflectance can be extracted at each spatial location independent from adjacent measurement locations. ${ }^{8}$

We have applied this handheld SFDS system in an established porcine burn wound model of graded burn severity. ${ }^{2}$ In this study, a specialized tool was used to create a series of graded burns on the back of a Yorkshire pig, $3 \mathrm{~cm}$ in diameter. The SFDS spectral imager measured the multiply scattered 
light from both burn region and adjacent tissue with $\sim 3 \mathrm{~nm}$ spectral resolution over several distinct longitudinal time points that allow us to compare preburn, acutely postburn, and at a couple of time points that correspond with those that are indicated as important time points for burn severity assessment (out to 3 days postburn). All experiments followed protocols reviewed and approved by the UC Irvine Institutional Animal Care and Use Committee (IACUC \#2015-3154).

Tissue absorption coefficient spectra are then extracted from these measurements and subsequently decomposed to identify all physiologic sources of absorption contrast present in the in vivo tissues, including those of hemoglobin breakdown products, over the wavelength range of 450 to $1000 \mathrm{~nm}$. From the spectral components identified by SFDS, we can demonstrate how the presence and dynamics of the methemoglobin in the wound healing process will impact the concentration estimation of other functional tissue parameters if not properly accounted for.

Using a validated spectral bandwidth emulation approach, we can transform SFDS data to mimic the same spectral qualities of multispectral imagers within the visible and near-infrared ranges. ${ }^{14}$ In this investigation, we consider the spectral performance of a commercially available, LED-based SFDI device: the OxImager RS, (Modulated Imaging, Inc.). Here, chromophore concentrations calculated from the emulated spectral data from the commercial instrument will be compared to those determined using the full spectral resolution of SFDS. This particular instrument was selected in this evaluation as it is has been used in several investigations of burn wounds and remains actively engaged in our ongoing studies. The spectral characteristics of this LED-based imager used for this evaluation have been published elsewhere. ${ }^{14}$

Figure 1 shows the evolution of tissue absorption within the burned tissue over time. Here, the spectra are derived from the average of a $\sim 1-\mathrm{cm}$ line ( $\sim 300$ pixels) within the center of the $3-\mathrm{cm}$ wide burn. Spectra recorded at earlier time points are included in visualization of later time points to help illustrate the shifts that occur in relative magnitude and shape of the absorption spectra. In the case of the 10-s burn (superficial), the spectral changes are slight, indicative of minimal tissue damage and wound healing response. There was a greater change in the 20-s burn (superficial partial thickness); however, the spectrum returned to its preburn shape and magnitude by $72 \mathrm{~h}$, potentially indicating a complete recovery. Both 30 and $40 \mathrm{~s}$ burns (deep partial and full thickness) not only fail to return to baseline by $72 \mathrm{~h}$, an additional, broad absorption feature becomes readily apparent by $24 \mathrm{~h}$. This feature is not replicated or explained by combinations of common tissue chromophores (i.e., oxy- and deoxyhemoglobin, water, melanin, lipids, etc.). Given the shape and location of absorption bands, this burninduced spectral feature correlates strongly with hemoglobin breakdown products, e.g., MetHb. To this end, we used the absorption spectrum for pure MetHb from Zijlstra et.al. ${ }^{18}$

Without the inclusion of MetHb in the spectral analysis, errors in the determination of several other tissue parameters will arise, as shown in Fig. 2. This figure depicts the spectral fits to the measured absorption spectra from the 30-s burn case (deep partial thickness burn). Here, the results of two least-squares fits are shown. The first fit result presumes oxyand deoxyhemoglobin, water, and melanin are the only absorbing constituents present in the tissue volume; the second fit result also includes MetHb in this spectral model. Using the normalized root mean square error as the cost function for a goodness of fit metric, including MetHb maintains a goodness of fit to within $85 \%$ of the measured data over all time points, but without its inclusion, the agreement can degrade to $\sim 56 \%$.

Inclusion of MetHb in the spectral fitting will not only improve the goodness of fit but also have significant impact on the quantification of other tissue chromophores and physiological parameters. Within the context of the four burn severities
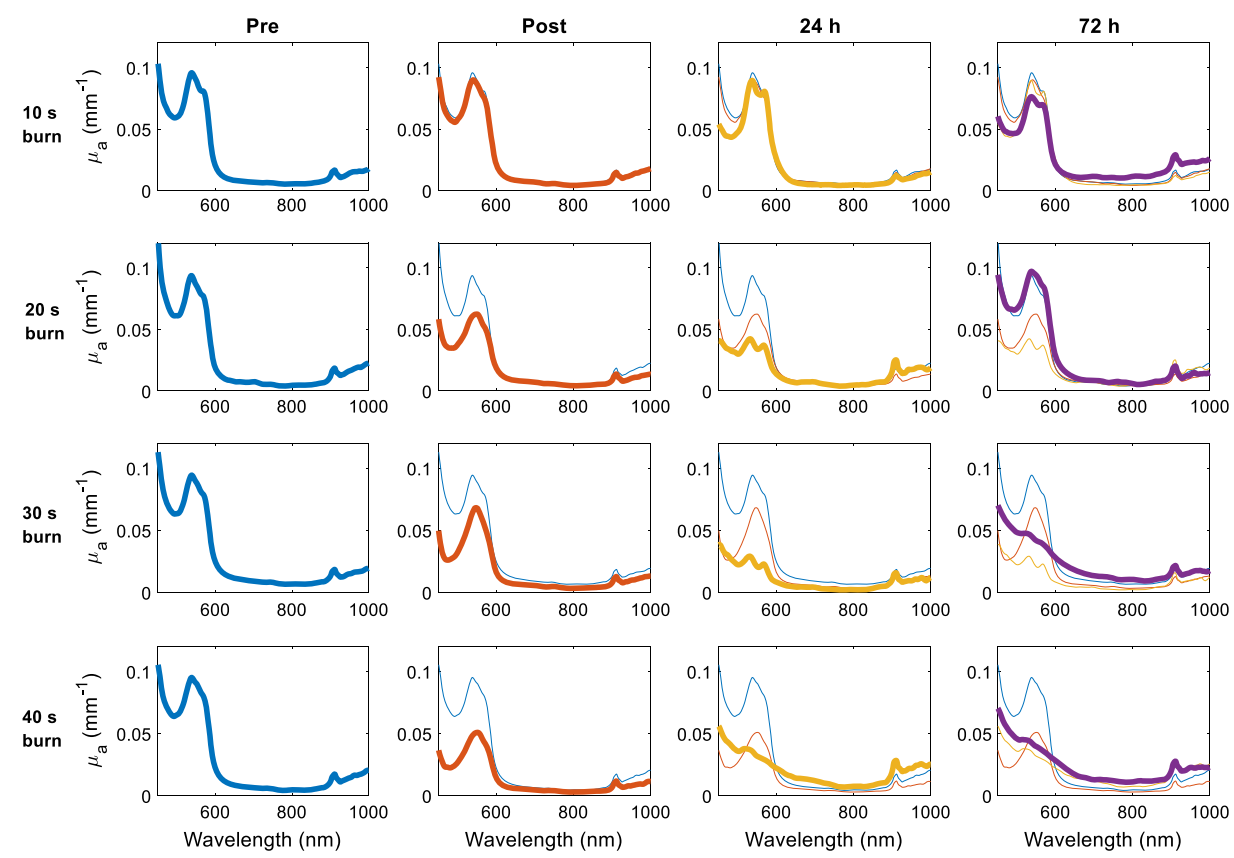

Fig. 1 Temporal evolution of absorption spectra as a function of burn severity (rows) and over discrete time points (columns). Thick lines are the absorption spectrum at a given time point, thin lines represent the spectra for that specific burn type from previous time points (for reference). 


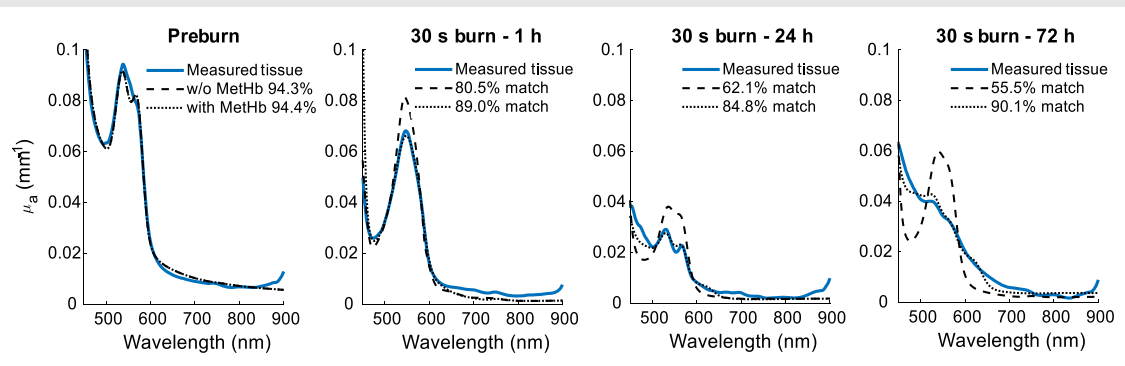

Fig. 2 Measured and fitted tissue absorption spectra for the $30 \mathrm{~s}$ (deep partial thickness) burn over time. Solid lines represent the measured absorption spectra. Dashed lines represent the linear least-squares fit produced when only $\mathrm{HbO}_{2}, \mathrm{Hb}$, water, and melanin are used as a spectral basis set. Dotted lines represent the fit when pure MetHb is added to the basis set.

investigated here, tissue oxygen saturation, $\mathrm{StO}_{2}$, is underestimated by as much as $20 \%$ within the first 3 days postburn and the tissue water fraction, is underestimated by as much as $78 \%$ when MetHb is not included in the fitting the measured SFDS absorption spectra. Largely, the magnitude of these underestimation errors directly correlate with increased concentration of MetHb within tissue volumes, hence these estimation errors increase with burn severity (i.e., 30 and $40 \mathrm{~s}$ burns), and with time (i.e., 24 and $72 \mathrm{~h}$ time points). It is worth noting that even in less severe burns, such as the case of the superficial partial thickness (20 s) burns, $\mathrm{StO}_{2}$ and water fraction may be underestimated by $12 \%$ and $8 \%$, respectively, $24 \mathrm{~h}$ after the injury.

Lastly, we have also determined that even with only eight spectral bands that span both visible and near-infrared wavelength regimes, a multispectral SFDI imager can account for MetHb, producing functional tissue parameters that agree with the high spectral resolution data (SFDS). Through emulating the OxImager's spectral profile with all the collected SFDS data, we can directly evaluate the impact that a reduced set of spectral bands and resolution may have on the calculation of tissue optical properties. In this case, this particular multispectral imager would determine $\mathrm{StO}_{2}$ within $3.9 \%$ and water fraction within $6.9 \%$ of the values calculated using all spectral content collected by SFDS. These results include all burn types and time points considered in this initial investigation.

While the goodness of fit metric, shown in Fig. 2, demonstrates that incorporating a pure MetHb spectrum in our fitting model significantly reduces residual features between measurement and model and supports the assertions of others, ${ }^{14,15}$ there are still some spectral regions where fitting is poor. Whereas pure methemoglobin has a distinct feature (shoulder) in its absorption near 630 to $640 \mathrm{~nm}$, the measured data do not indicate any such shoulder, but rather shows a smooth, featureless slope through that spectral range. One possible explanation for this discrepancy is that the additional absorption feature measured in tissue is not pure MetHb, but rather a continuum of transitional heme products that are also evolving over time. As the molecular structure is progressively being altered, absorption bands could shift and/or broaden and thereby result in a measured absorption spectrum that represent the larger, general shape of MetHb, yet wash out the finer, distinct features. Further investigation into this possibility is ongoing. Interestingly, initial attempts in fabricating hemoglobin-based tissue simulating phantoms from freeze-dried bovine hemoglobin have resulted in similar washed out, MetHb-like absorption features. ${ }^{19,20}$

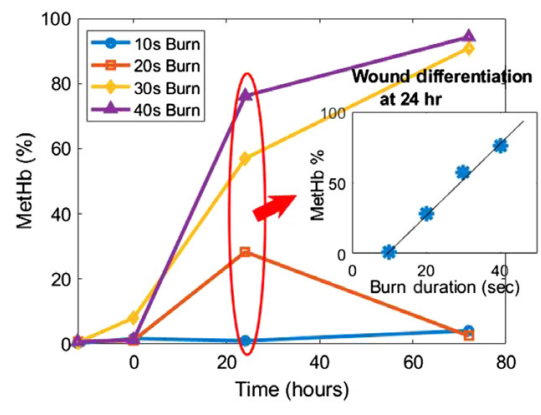

Fig. 3 Methemoglobin concentration over time. Inset figure shows the positive correlation of the calculated concentration to burn severity (time duration of burn injury) after $24 \mathrm{~h}$.

In addition to the identification of spectral signatures from MetHb present in burn wound tissue and how that may impact reliable assessment of tissue function, quantification of this chromophore may also hold some diagnostic value on its own, as others have suggested. ${ }^{15}$ Figure 3 shows the changes in calculated MetHb concentrations (plotted in terms of volume fraction) over time for each burn type. While little MetHb was detected within the first hour of the burn, there was a dramatic spread of concentrations at the $24-\mathrm{h}$ time point. Plotting the $24-\mathrm{h}$ concentration values as a function of burn severity showed a surprisingly linear correlation. Since it is drawn from such a small data set, this result is merely anecdotal. However, it may suggest that quantification of MetHb may provide additional and complementary information to assess and characterize burn wound severity. While parameters such as $\mathrm{StO}_{2}$ and water fraction (edema) may indicate the tissues functional response to the injury, MetHb may indicate a measure of the amount of damage. The combination of these parameters may present unique opportunities to advance burn wound assessment toward personalized medicine where it is critical to know not just how much damage has occurred within the wound, but whether the patient has initiated a wound healing response and whether that response will be sufficient to heal the wound on its own.

In conclusion, we have demonstrated that additional hemoglobin species will arise in tissue from burn wound injuries and dynamically vary in concentration during the wound healing process. Based on the spectral characterization from in vivo burn wound measurements, these absorption features correlate to hemoglobin breakdown products, e.g., MetHb. Additionally, we have also demonstrated that it is important to include MetHb in any multispectral image analysis in order to produce robust 
estimates of other tissue parameters, such as $\mathrm{StO}_{2}$ and water fraction.

\section{Disclosures}

Dr. Durkin has a financial interest in Modulated Imaging, Inc., which developed the Reflect RS ${ }^{\mathrm{TM}}$. However, Dr. Durkin does not participate in the management of Modulated Imaging and has not shared these results with that company. Conflicts of interest have been disclosed and carefully managed in accordance with the University of California and NIH policies. The other authors have no financial interests or commercial associations that might pose or create a conflict of interest with the information presented in this article.

\section{Acknowledgments}

We thankfully recognize support from NIGMS grant R01GM108634-01A which enabled the use of the OxImager RS and collection of porcine data. We also recognize support from the NIH, including NIBIB P41EB015890 (A Biomedical Technology Resource) SFDS device fabrication was facilitated via, a triumvirate grant from the UCI Institute for Clinical and Translational Sciences. The content is solely the responsibility of the authors and does not necessarily represent the official views of the NIGMS, NIBIB, or NIH. In addition, this material is based on technology development supported in part by the Air Force Office of Scientific Research under award number FA9550-17-1-0193. Any opinions, findings, and conclusions or recommendations expressed in this material are those of the authors and do not necessarily reflect the views of the United States Air Force. We also thank the Arnold \& Mabel Beckman Foundation.

\section{References}

1. J. Q. Nguyen et al., "Spatial frequency domain imaging of burn wounds in a preclinical model of graded burn severity," J. Biomed. Opt. 18(6), 066010 (2013).

2. A. Ponticorvo et al., "Quantitative assessment of graded burn wounds in a porcine model using spatial frequency domain imaging (SFDI) and laser speckle imaging (LSI)," Biomed. Opt. Express 5(10), 3467-3481 (2014).

3. A. J. Singer and R. A. Clark, "Cutaneous wound healing," N. Engl. J. Med. 341(10), 738-746 (1999).

4. L. F. Rose et al., "The burn wound microenvironment," Adv. Wound Care (New Rochelle) 5(3), 106-118 (2016).
5. L. L. Randeberg et al., "Methemoglobin formation during laser induced photothermolysis of vascular skin lesions," Lasers Surg. Med. 34(5), 414-419 (2004).

6. L. L. Randeberg et al., "A novel approach to age determination of traumatic injuries by reflectance spectroscopy," Lasers Surg. Med. 38(4), 277-289 (2006).

7. L. L. Randeberg, E. L. Larsen, and L. O. Svaasand, "Characterization of vascular structures and skin bruises using hyperspectral imaging, image analysis and diffusion theory," J. Biophotonics 3(1-2), 53-65 (2010).

8. D. J. Cuccia et al., "Quantitation and mapping of tissue optical properties using modulated imaging," J. Biomed. Opt. 14(2), 024012 (2009).

9. D. M. Burmeister et al., "Utility of spatial frequency domain imaging (SFDI) and laser speckle imaging (LSI) to non-invasively diagnose burn depth in a porcine model," Burns 41(6), 1242-1252 (2015).

10. A. Ponticorvo et al., "Quantitative long-term measurements of burns in a rat model using spatial frequency domain imaging (SFDI) and laser speckle imaging (LSI)," Lasers Surg. Med. 49(3), 293-304 (2017).

11. T. T. Nguyen et al., "Novel application of a spatial frequency domain imaging system to determine signature spectral differences between infected and noninfected burn wounds," J. Burn Care Res. 34(1), 44-50 (2013).

12. C. Poon et al., "Early assessment of burn severity in human tissue ex vivo with multi-wavelength spatial frequency domain imaging," Toxicol. In Vitro 52, 251-254 (2018).

13. A. Ponticorvo et al., "Evaluating clinical observation versus spatial frequency domain imaging (SFDI), laser speckle imaging (LSI) and thermal imaging for the assessment of burn depth," Burns (2018).

14. R. B. Saager et al., "Method using in vivo quantitative spectroscopy to guide design and optimization of low-cost, compact clinical imaging devices: emulation and evaluation of multispectral imaging systems," J. Biomed. Opt. 23(4), 046002 (2018).

15. K. M. Cross, "Assessment of tissue viability in acute thermal injuries using near infrared point spectroscopy," University of Toronto (2010).

16. R. B. Saager, D. J. Cuccia, and A. J. Durkin, "Determination of optical properties of turbid media spanning visible and near-infrared regimes via spatially modulated quantitative spectroscopy," J. Biomed. Opt. 15(1), 017012 (2010).

17. R. B. Saager et al., "Portable (handheld) clinical device for quantitative spectroscopy of skin, utilizing spatial frequency domain reflectance techniques," Rev. Sci. Instrum. 88(9), 094302 (2017).

18. W. G. Zijlstra, A. Buursma, and W. P. Meeuwsen-van der Roest, "Absorption-spectra of human fetal and adult oxyhemoglobin, de-oxyhemoglobin, carboxyhemoglobin, and methemoglobin," Clin. Chem. 37(9), 1633-1638 (1991).

19. I. Fredriksson et al., "Evaluation of a pointwise microcirculation assessment method using liquid and multilayered tissue simulating phantoms," J. Biomed. Opt. 22(11), 115004 (2017).

20. R. B. Saager et al., "From theory to practice: the broadening role of polydimethylsiloxane phantoms as an intermediary between model validation and instrument performance testing," Proc. SPIE 9700, 97000G (2016). 